\title{
Type I polyglandular syndrome patient presenting with metabolic encephalopathy
}

\author{
NP Premawardana ${ }^{1}$, KD Pathirana ${ }^{2}$ \\ ${ }^{1}$ Registrar in Medicine, Professorial Medical Unit, Teaching Hospital, Karapitiya, Galle. \\ ${ }^{2}$ Consultant Neurologist, Senior Lecturer, Department of Medicine, Faculty of Medicine, Galle.
}

\section{Introduction}

When two or more endocrine glands and other non endocrine immune disorders are present, the polyglandular autoimmune (PGA) syndromes should be considered. There are two major types of polyglandular autoimmune syndromes [1].

Type I polyglandular failure is characterised primarily by adrenal insufficiency, m u c o c u t a n e u s c a n d idiasis, hypoparathyroidism, and an array of other endocrine and non-endocrine disorders which occur with variable frequency [2].
PGA type I requires two of the three primary components for diagnosis. At the onset only one organ may be involved, but the number increase with time so that patients eventually manifest two to five components of the syndrome $[1,2]$.

Type II polyglandular failure (Schmidt syndrome) shares the high prevalence of adrenal insufficiency, but differs from the type I syndrome in that common features include autoimmune thyroid disease and insulin dependent diabetes mellitus. The onset of type II PGA is usually later than in type I, occurring primarily in adults [2]. 
We describe an unusual case of polyglandular syndrome type I brought to the hospital with a state of unconsciousness due to metabolic encephalopathy.

\section{Case Report}

\section{History and Examination}

A man in his early thirties found unconscious and brought to the emergency treatment unit of Teaching Hospital, Karapitiya by the police. He developed a tonic clonic seizure lasting for 3 minutes which resolved spontaneously. Though he regained consciousness within next few hours, he remained drowsy and confused.

Next day he became irritable and aggressive attacking the caring nursing staff. He developed fits again and went into a semiconscious state. $\mathrm{He}$ had fluctuating level of consciousness for next few days, but no fits. He also had watery diarrhea lasted for two days which settled with no specific treatment. There was no significant illness in the past but family members admitted that he had been an alcohol addict.

At the time of admission he was drowsy. Glasgow coma scale was 10/15. Pupils were equal in size with sluggish response to light. $\mathrm{He}$ was dehydrated but not febrile or pale. He had bilateral cataract. He had no lymphadenopathy. He had normal secondary sexual characteristics. He had whitish plaques over the buccal mucosa and tongue but no skin lesions.

Chvostek's and Trousseau's signs were negative. He had generalised rigidity with neck stiffness but no cogwheel rigidity. Muscle power was grade $4 / 5$ in both upper and lower limbs. Plantar responses were flexor bilaterally. Blood pressure was $100 / 60 \mathrm{mmHg}$, with no postural drop. Rest of the cardiovascular system and also the respiratory system examination and the abdominal examination were normal. Digital rectal examination revealed mild prostatomegaly.

\section{Investigations}

His haemoglobin concentration was $10.6 \mathrm{~g} / \mathrm{dL}$. There was polymorphoneuclear leucocytosis.
His blood urea was $70 \mathrm{mg} / \mathrm{dL}$. He had hypokaleamia $(2.8 \mathrm{mmol} / \mathrm{L})$. Urinary electrolytes revealed $\mathrm{Na}^{+}$of $75 \mathrm{mmol} / \mathrm{L}$ and $\mathrm{K}^{+}$of $15 \mathrm{mmol} / \mathrm{L}$. Electrocardiography showed prolonged QT interval with abnormal U waves. Electroencephalography revealed generalised increased theta and delta activity with EMG artifact in the frontal montages, compatible with metabolic encephalopathy (Figure 1). Fasting blood sugar was $95 \mathrm{mg} / \mathrm{dL}$.

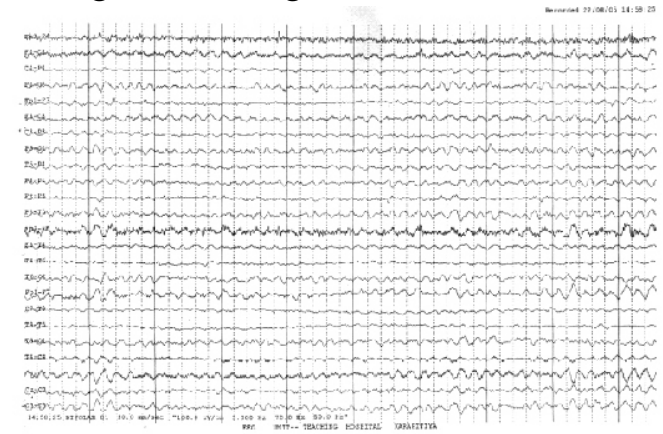

Figure 1 - Electroencephalography

CT scan of brain showed bilateral pathological basal ganglion and cerebellar calcification (Figure 2). He had low serum ionized calcium of $0.92 \mathrm{mmol} / \mathrm{L}(1.12-1.32 \mathrm{mmol} / \mathrm{L})$ and with high serum inorganic phosphorus of $7.20 \mathrm{mg} / \mathrm{dL}$ $(2.50-4.50 \mathrm{mg} / \mathrm{dL})$. Parathyroid hormone level was $<3.00 \mathrm{pg} / \mathrm{mL}(07-53 \mathrm{pg} / \mathrm{mL}$ with a median of $32 \mathrm{pg} / \mathrm{mL}$ ).

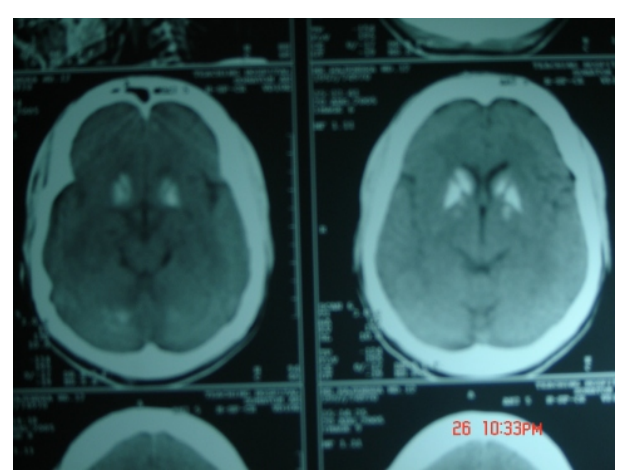

Figure 2 - CT Scan Brain (Non contrast)

\section{Discussion}

Hypocalcaemia with bilateral basal ganglion calcification, hyperphosphataemia and low serum parathyroid hormone (PTH) level confirms the diagnosis of hypoparathyroidism $[3,4]$. 
These features are shared by both types of hypoparathyroidism, acquired and hereditary [3]. He had no surgical scars to suggest that he had undergone previous thyroidectomy or any other surgery in the neck. Basal ganglia calcification and extra pyramidal syndromes are common and earlier in onset in hereditary hypoparathyroidism [3].

Although pseudohypoparathyroidism can cause similar CT appearance, it is unlikely in this case due to absence of other skeletal features and the low PTH levels. Hyperplasia of the parathyroids, a response to PTH resistance in pseudohypoparathyroidism, causes elevation of PTH level [3,5].

Since idiopathic hypoparathyroidism can be part of autoimmune polyendocrine syndrome he was screened for other endocrine abnormalities to prevent illness associated with delayed diagnosis of additional autoimmune diseases [6].

Short Synacthen test was carried out by administration of 25 units $(0.25 \mathrm{mg})$ of cosyntropin intravenously and measurement of plasma cortisol concentrations on 0,30 and 60 minutes later. His serum cortisol concentration did not show any increase confirming the adrenal insufficiency. Thyroid profile, FSH and LH levels were normal.

Hypokalemia was a result of increased net loss of $\mathrm{K}^{+}$due to diarrhoea. Urinary $\mathrm{K}^{+}$was $<20 \mathrm{mmol} / \mathrm{L}$ indicating renal compensation to gastrointestinal loss. Elevated blood urea was due to dehydration and became normal with correction of volume. Ultrasound scan revealed no renal parenchymal disease.

He was treated with Phenytoin Sodium 100mg twice daily on admission to control fits. $1 \alpha$ calciferol $0.25 \mathrm{mg}$ daily and calcium lactate 2 tablets twice daily was commenced when investigation results confirmed the hypocalcaemia. Within 72 hours of starting calcium supplementation he became conscious and rational. He was started on oral hydrocortisone $30 \mathrm{mg}$ daily after short Synacthen test.

Primary hypoparathyroidism, adrenal insufficiency with mucocutaneous lesions suggestive of candidiasis favours the diagnosis of polyglandular syndrome type I [7]. Most patients initially present with oral candidiasis in childhood. It is poorly responsive to treatment and relapses frequently. Chronic hypoparathyroidism usually occurs before adrenal insufficiency. The endocrine components, including adrenal insufficiency and hypoparathyroidism, may not develop until the forth decade, making continued surveillance necessary $[1,7]$.

PGA type I is usually inherited as an autosomal recessive trait. The responsible gene, designated as either APECED (Autoimmune polyendocrinopathy - candidiasis-ectodermal dystrophy) or AIRE (Autoimmune regulator) encodes a transcription factor that is expressed in the thymus and lymph- nodes; a variety of different mutations have been reported $[1,4]$.

The clinical manifestation of adrenal insufficiency often develops slowly and may be difficult to detect. It can be fatal if not diagnosed and treated appropriately. Thus, prospective screening should be performed routinely in all patients and family members at risk for PGA type I and II.

He recovered fully and subsequently worked as a labourer in the janitorial service in the hospital. Later he reverted back to alcohol abuse and lost for follow up.

\section{References}

1. Steven I. Sherman, Robert F. Gagel. Disorders affecting multiple organ systems. Harrison's Principles of Internal Medicine - $16^{\text {th }}$ edition (page 2231-2238).

2. J.L. Jameson. Principles of hormone action. Oxford Text Book of Medicine $-3^{\text {rd }}$ edition

3. John T. Potts, Jr Diseases of parathyroid gland and hyper and hypocalcaemia. Harrison's Principles of Internal Medicine $-16^{\text {th }}$ edition (page 2249-2268).

4. Basic and Clinical Endocrinology ( $4^{\text {th }}$ edition $)$ Francis S. Greenspan, John Baxter. (page 242).

5. Elaine Murphy, Graham R Williams. Hypocalcaemia. Medicine International 33:12 page 55-57.

6. George S. Elsenbarth and Peter A. Gottlieb. Autoimmune poly endocrine syndrome. New England Journal of Medicine 2004; 350: 2068-79.

7. William's textbook of Endocrinology. ( $9^{\text {th }}$ edition $)$ Jean D. Wilson, Daniel Foster, Henry M. Kronenberg, P. Reed Larsen ( page 1657) 